\title{
Caracterização e Análise das Redes de Colaboração Científica dos Bolsistas de Produtividade em Pesquisa do CNPq
}

\author{
Thiago M. R. Dias ${ }^{1}$, Tales H. J. Moreira ${ }^{1}$, Patrícia M. Dias ${ }^{2}$ \\ ${ }^{1}$ Centro Federal de Educação Tecnológica de Minas Gerais - CEFET-MG \\ ${ }^{2}$ Universidade do Estado de Minas Gerais - UEMG \\ thiagomagela@cefetmg.br, tales.info@gmail.com, patrícia.dias@uemg.br
}

\begin{abstract}
Studies of scientific collaboration networks have for some time been receiving attention from analysts in various fields of knowledge because of their potential to identify how groups of researchers have collaborated in their research. Such studies make it possible to identify with the aid of network analysis metrics, how networks are formed, how they evolve over time, and how they are structured. In this research, a characterization and analysis of the scientific collaboration networks of the CNPq Research Productivity Grantees is presented. As a result, it is presented as the main Brazilian researchers, who receive incentive for excellence in their research has collaborated with each other.
\end{abstract}

Resumo. Estudos sobre as redes de colaboração científica vêm a algum tempo recebendo atenção de analistas de diversas áreas do conhecimento devido seu potencial de identificar como grupos de pesquisadores têm colaborado em suas pesquisas. Tais estudos possibilitam identificar com o auxílio de métricas de análises de redes, como as redes são formadas, como evoluem ao longo do tempo e como são estruturadas. Neste trabalho é apresentada uma caracterização e análise das redes de colaboração científica dos Bolsistas de Produtividade em Pesquisa do CNPq. Como resultado, é apresentado como os principais pesquisadores brasileiros, que recebem incentivo pela excelência em suas pesquisas tem colaborado entre si.

\section{Introdução}

Com o crescimento da divulgação científica impulsionada principalmente pela disponibilidade e acesso imediato a repositório de dados de publicações científicas, uma nova geração de serviços disponíveis principalmente na Web está mudando a forma de divulgar e disponibilizar a produção científica e tecnológica. Existe, atualmente, uma tendência que reforça a troca de informações e a colaboração entre as pessoas. A forte relação entre os domínios científico e socioeconômico tem gerado um interesse crescente pela compreensão dos mecanismos que norteiam as atividades científicas, sendo possível apontar diversos trabalhos que analisam aspectos específicos como as características da linguagem e dos discursos empregados na comunicação científica (HOFFNAGEL, 2009) ou, ainda, a relação de colaboração entre pesquisadores e grupos de pesquisa (DING, 2011; REVOREDO et al., 2012; STROELE; ZIMBRÃO; SOUZA, 2012). 
Nesse cenário, a Bibliometria (ARAÚJO, 2006), que possui grande relação com a Cientometria (SILVA; HAYASHI; HAYASHI, 2011), se destaca como uma das principais ciências métricas de análise de conteúdo, podendo ser aplicada a fontes de dados científicos com o intuito de se obter informações quantitativas sobre publicações (PRITCHARD, 1969). Diante disso, é possível analisar dados de publicações científicas com o objetivo de identificar as tendências e o crescimento do conhecimento em diversas áreas, prever padrões de pesquisa, observar a dispersão do conhecimento científico, auxiliar políticas de auxílio à pesquisa e entender como ocorre a evolução científica de uma determinada área do conhecimento ou de grupos de pesquisadores.

A produção de indicadores bibliométricos considerados mais representativos tornou-se realidade concreta na s últimas décadas do século XX. Os principais motivos para a proposta de tais indicadores devem-se à construção, manutenção e informatização de repositórios de dados científicos (MUGNAINI; JANNUZZI; QUONIAM, 2004). Por sua vez, um dos fatores que têm motivado os estudos de avaliação da produção científica, principalmente com a adoção de métricas bibliométricas, tem sido a busca pela excelência em áreas de pesquisa e, também, a competição pelos recursos financeiros das agências de fomento (LOPES, 2012).

Nos últimos anos, além da análise bibliométrica de publicações científicas, diversos outros estudos têm procurado compreender como a ciência tem evoluído e como a colaboração científica ocorre. Diante disso, técnicas baseadas em análises de redes surgem como uma alternativa para verificar esse fenômeno. De modo geral, uma rede pode ser caracterizada como um grafo, que consiste de um conjunto de nós (vértices) e ligações (arestas) entre os nós (SZWARCFITER, 1986). Essas ligações podem ser direcionadas ou não e, opcionalmente, podem ainda ter um peso associado.

No domínio científico, um exemplo de uma rede social é a de colaboração científica, que pode ser observada como uma rede na qual os nós correspondem aos autores de publicações científicas e as arestas correspondem à relação de coautoria. Nesse tipo de rede, as arestas podem ou não ser ponderadas. A adição de um peso representa o número de trabalhos em que os autores relacionados pela aresta considerada participaram conjuntamente. Dessa forma, a intensidade dos relacionamentos presentes em uma rede de colaboração científica é medida pelo número de colaborações entre um par de autores. A presença do peso é útil para representar, por exemplo, a afinidade e os interesses comuns entre dois autores da rede (SONNENWALD, 2007).

Com a modelagem e caracterização das redes de colaboração científica, é possível aplicar diversas técnicas que permitem entender como essas redes são estruturadas, fornecendo assim subsídios para diversos estudos como predição de vínculos entre pesquisadores, recomendação de especialistas e identificação de grupos de pesquisa.

Neste contexto, este trabalho visa responder a seguinte questão: Como ocorre a colaboração científica entre os principais pesquisadores do Brasil ?

Atualmente no Brasil, pesquisadores com elevada capacidade de pesquisa são reconhecidos com o recebimento de uma bolsa de produtividade, paga pelo Conselho Nacional de Desenvolvimento Científico e Tecnológico (CNPq) pela excelência em suas pesquisas. A bolsa de produtividade em pesquisa do CNPq é destinada aos pesquisadores 
que se destaquem entre seus pares na realização de pesquisas nas áreas científicas e tecnológica. Dessa forma, o CNPq oferece um estímulo constante aos pesquisadores de excelência no país, valorizando a qualidade, o aprofundamento e a possível aplicação de novos estudos (SANTOS, 2016).

Diante disso, este trabalho apresenta um estudo sobre as redes de colaboração científica dos Bolsistas de Produtividade em Pesquisa do CNPq, baseado em análises de redes sociais que possibilitam compreender como ocorre a colaboração científica do conjunto, e como estão colaborando os pesquisadores brasileiros de excelência nas diversas modalidades de bolsas. Para isso, são analisados dados dos currículos cadastrados na Plataforma Lattes do $\mathrm{CNPq}$ responsável por armazenar informações curriculares da comunidade científica nacional. Atualmente a Plataforma Lattes é considerada uma importante fonte de dados sobre a produção científica brasileira (LANE, 2010).

O restante deste trabalho está organizado da seguinte forma. A Seção 2 apresenta alguns trabalhos relacionados ao conteúdo do artigo, a Seção 3 descreve como foi construído e processado o conjunto de dados utilizados, a Seção 4 apresenta os resultados das análises realizadas e a Seção 5 apresenta as considerações finais e propostas para novos estudos.

\section{Trabalhos Relacionados}

Barabási et al. (2002) apresentam um estudo das redes de colaboração científica nas áreas de Neurociência e Matemática entre os anos de 1991 e 1998. Os autores concluíram que a rede evolui a partir do momento em que novos nós e vínculos entre os nós existentes são incluídos. E que esta evolução segue um processo denominado preferential attachment, em que novos nós tendem a criar seu primeiro vínculo com nós que possuem grande número de vínculos. Como resultado, autores experientes tendem a aumentar seu número de colaboradores, com maior frequência do que autores novatos.

Para Revoredo et al. (2012), redes como as de comunidades científicas de formação recente possuem poucos parâmetros para classificação de assuntos de interesse e pouco entendimento tanto da existência como o potencial de relações de colaboração. Nestas comunidades, a compreensão de sua composição e tendências de interesse se beneficia de técnicas de descoberta de conhecimento a partir de seus artefatos principais de produção, as publicações.

Segundo Stroele, Zimbrão e Souza (2012), a análise de redes de colaboração científica possibilita identificar como os grupos de pesquisadores e centros de estudos estão desenvolvendo seus trabalhos, verificar e entender qual o grau de envolvimento entre os pesquisadores de determinados grupos, de determinadas áreas do conhecimento, de instituições de ensino e pesquisa, e também permitir a indicação de padrões de colaboração que poderiam proporcionar um grande avanço na área, permitindo melhorias na comunicação e colaboração de toda a comunidade científica.

Mena-Chalco et al. (2014) utilizam dados dos currículos da Plataforma Lattes para identificar e caracterizar a rede de colaboração de pesquisadores brasileiros. O trabalho objetivou extrair os dados de currículos cadastrados na Plataforma Lattes, identificar automaticamente a colaboração baseada em informações bibliométricas, produzindo uma rede de colaboração, e aplicar métricas baseadas em análise topológica para compreender como ocorre a interação entre os pesquisadores. 
No trabalho de Digiampietri et al. (2017), é apresentada uma análise da evolução, impacto e formação de redes nos cinco anos do BraSNAM (Brazilian Workshop on Social Network Analysis and Mining). Os autores analisaram baseado nas cinco primeiras edições do evento, a produção bibliográfica e a evolução da rede de coautoria. No estudo das redes de coautoria os trabalhos publicados em cada edição foram utilizados para a caracterização de cada uma das redes. Foi observado uma evolução do evento que anualmente tem atraído novos pesquisadores, contribuindo para a expansão e consolidação do BraSNAM.

Como diferencial deste trabalho em relação aos citados anteriormente, este estudo visa apresentar como ocorre a colaboração científica dos principais pesquisadores brasileiros considerando todo o conjunto de Bolsistas de Produtividade em Pesquisa do $\mathrm{CNPq}$, bem como, por níveis das bolsas que recebem como auxílio.

\section{Materiais e Métodos}

Para a análise da colaboração científica do conjunto de Bolsistas de Produtividade em Pesquisa do $\mathrm{CNPq}$, foram utilizados dados extraídos de seus currículos cadastrados na Plataforma Lattes.

Uma grande parte dos editais de financiamento de projetos de pesquisa realizados por agências de amparo à pesquisa, por instituições de ensino, bem como pelo próprio CNPq, utilizam dados registrados nos currículos cadastrados na Plataforma Lattes dos proponentes como uma das formas de avaliação das propostas. Isto passou a ser um grande incentivo para que os pesquisadores mantenham seus currículos com informações atualizadas, tornando a Plataforma Lattes uma fonte extremamente rica para análise da produção científica brasileira.

Para o processo de coleta e tratamento dos dados foi utilizado um framework denominado LattesDataXplorer (Dias, 2016). Ele é responsável por englobar todo um conjunto de técnicas e métodos para realizar a extração de todo o conjunto de currículos cadastrados na Plataforma Lattes, e realizar diversos processos para o tratamento, seleção e análises dos dados curriculares (Figura 1).

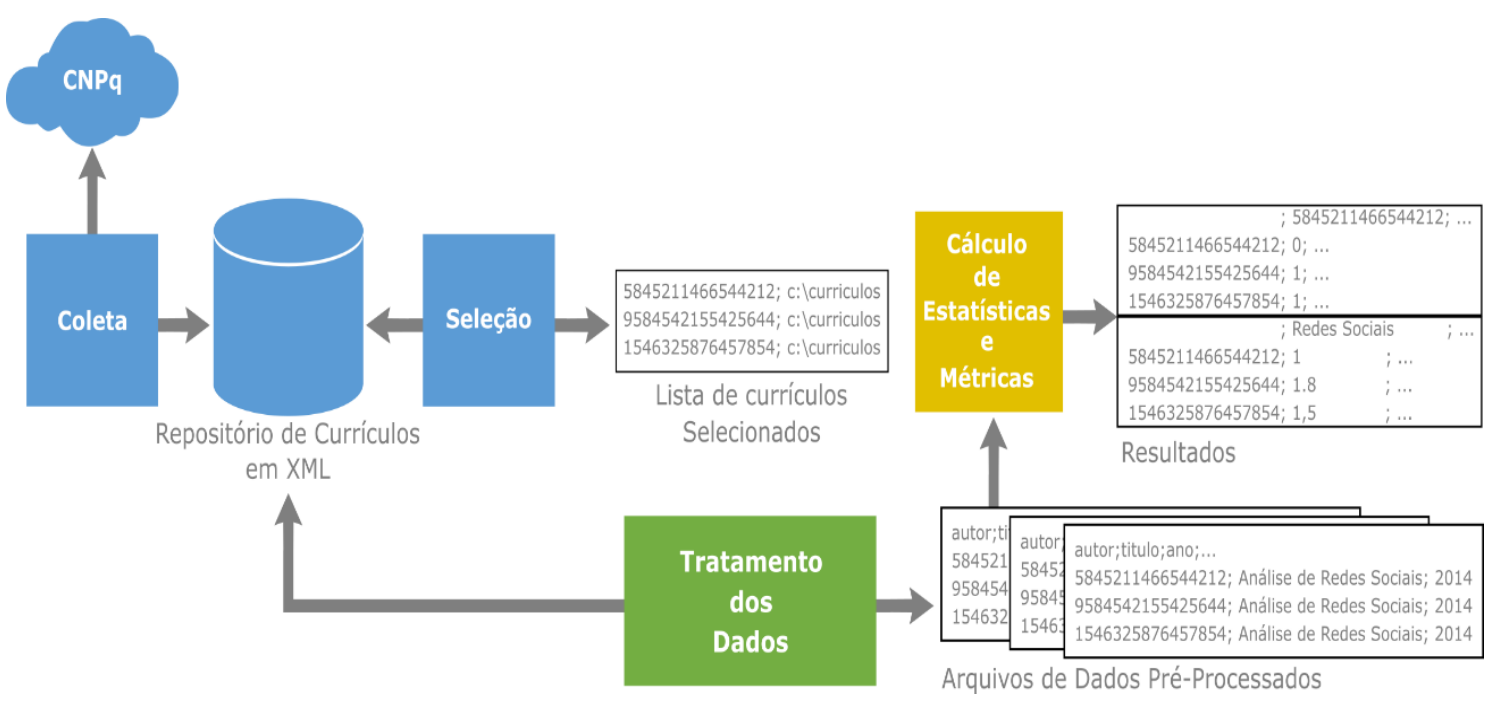

Figura 1. LattesDataXplorer, framework para extração e tratamento de dados curriculares da Plataforma Lattes (Dias, 2016). 
O processo de coleta de todos os dados curriculares da Plataforma Lattes é dividido em três componentes que objetivam minimizar o custo computacional: 1) extração de URLs, que é responsável por extrair as referências únicas para todos os currículos cadastrados e desta forma possibilitar o acesso individual a cada currículo, 2) extração de Identificadores (Ids) e Data, que visa acessar cada currículo e extrair o seu identificador individual, bem como a data de última atualização, 3) extração de currículos, que é responsável por extrair e armazenar os currículos (em formato XML) cuja data de atualização na Plataforma Lattes seja divergente da data de atualização do currículo armazenado localmente.

Todas essas etapas se fazem necessárias, já que o ideal é manter os dados curriculares atualizados com a maior frequência possível, possibilitando a realização de análises com dados atualizados, tendo em vista que, com a estratégia adotada, não se faz necessário extrair todo o repositório de dados a cada nova extração. Além dos componentes responsáveis pelo processo de coleta dos dados, e considerando a necessidade de análises de grupos específicos, como por exemplo, dos Bolsistas de Produtividade em Pesquisa do CNPq, um componente denominado Seleção, caracterizase como importante mecanismo no contexto deste trabalho.

O componente de Seleção utiliza a linguagem de consulta XPath (XML Path Language) para pesquisa no repositório local de currículos e posterior geração do conjunto. A linguagem XPath permite construir expressões que vão processar e percorrer um documento XML de forma similar ao uso de expressões regulares. Logo, é possível agrupar um conjunto de currículos com base em parâmetros desejados. Diante disso, foi possível identificar no repositório local que dentre os 5.620.451 currículos extraídos em 02/2018, um conjunto de 14.475 indivíduos possuem registrado em seus currículos que eles são Bolsista de Produtividade em Pesquisa do CNPq em alguma modalidade de bolsa. Logo, os dados contidos nos currículos deste conjunto de indivíduos foram analisados, sendo apresentada uma visão geral deste conjunto, e posteriormente, caracterizadas as redes de colaboração científica para cada modalidade de bolsa, além da rede geral, contendo todos os bolsistas.

\section{Resultados}

Para análises da colaboração científica dos pesquisadores de excelência no Brasil, considerou-se o conjunto de Bolsistas de Produtividade em Pesquisa do CNPq. Este grupo de indivíduos que, em sua maioria, tem atuado em pesquisas, seja em instituições de ensino seja em institutos de ciência e tecnologia, ainda é responsável pela formação dos alunos nos principais programas de pós-graduação no Brasil. Com isso, ressalta-se que o conjunto de indivíduos analisado neste trabalho, apesar de englobar uma pequena quantidade de indivíduos, compreende grande parte dos principais pesquisadores em atuação no Brasil.

O sistema de bolsas Produtividade em Pesquisa do CNPq encontra se estruturado em modalidades, iniciando se pela modalidade 2, em que o bolsista deve ser portador de título de doutor há pelo menos três anos na ocasião da implementação da bolsa. Já na modalidade 1, ele deve possuir no mínimo, oito anos de doutorado na ocasião da implementação da bolsa. Essa modalidade encontra se dividida em quatro subníveis (A, $\mathrm{B}, \mathrm{C}$ e D) estabelecidas em bases comparativas com a comunidade científica da área e 
tendo por base os dados dos últimos dez anos, incluindo a capacidade de formação contínua de recursos humanos, especialmente orientações de pós-graduação stricto sensu. A distribuição do conjunto selecionado nas modalidades de bolsas pode ser visualizada na Figura 2.

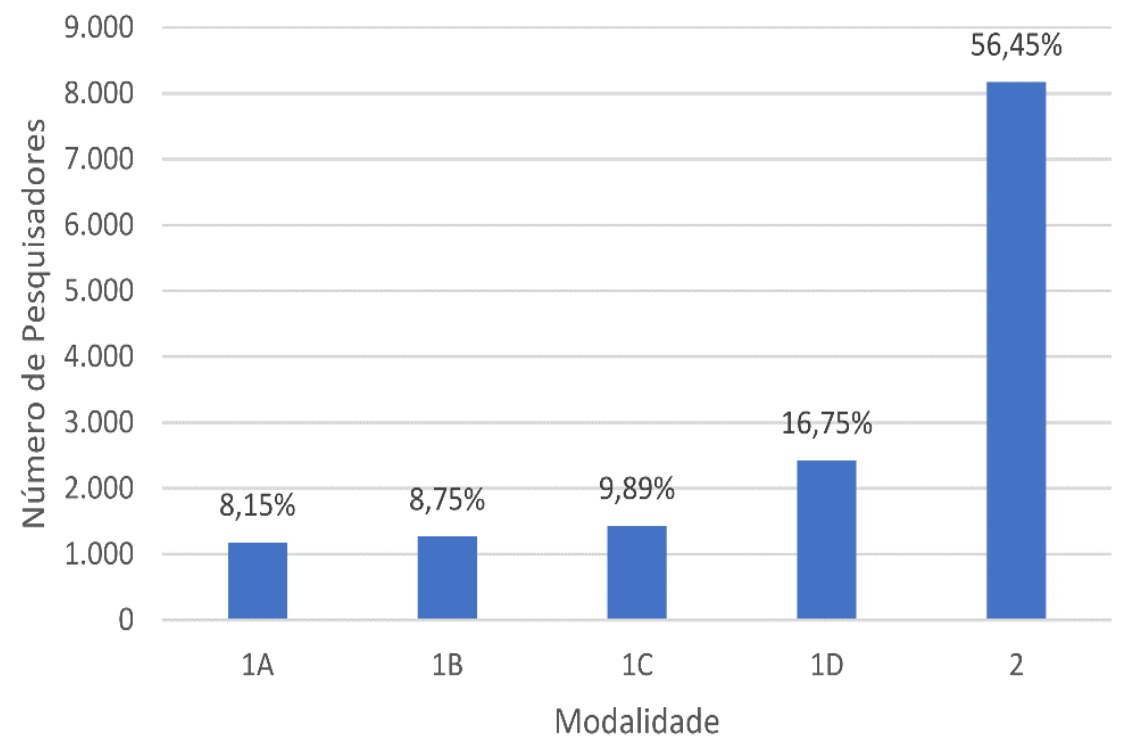

Figura 2. Distribuição dos bolsistas nas modalidades de bolsas.

Como pode ser observado, a modalidade 2 concentra a maior quantidade de bolsistas. Consequentemente, os outros pesquisadores que representam aproximadamente $44 \%$ do conjunto, são distribuídos nas modalidades superiores em que, a quantidade de indivíduos reduz em cada modalidade, à medida que o nível da bolsa aumenta.

Destaca-se que obrigatoriamente os bolsistas ingressam na modalidade 2, e posteriormente, podem progredir para as modalidades de níveis mais altos. Logo, a modalidade 2 tende naturalmente a concentrar uma maior quantidade de bolsistas, tendo em vista que é a modalidade de entrada para todos os pesquisadores.

No contexto deste trabalho, que visa caracterizar as redes de colaboração científica do conjunto de bolsistas, a produção científica é o principal elemento de estudo. Foi identificado que em ambas as modalidades de bolsas, os bolsistas tendem a divulgar sua produção científica preferencialmente em anais de congressos e em periódicos. A Figura 3 apresenta o total de publicações de todos os bolsistas, em ambos os meios de divulgação por modalidade de bolsas. 


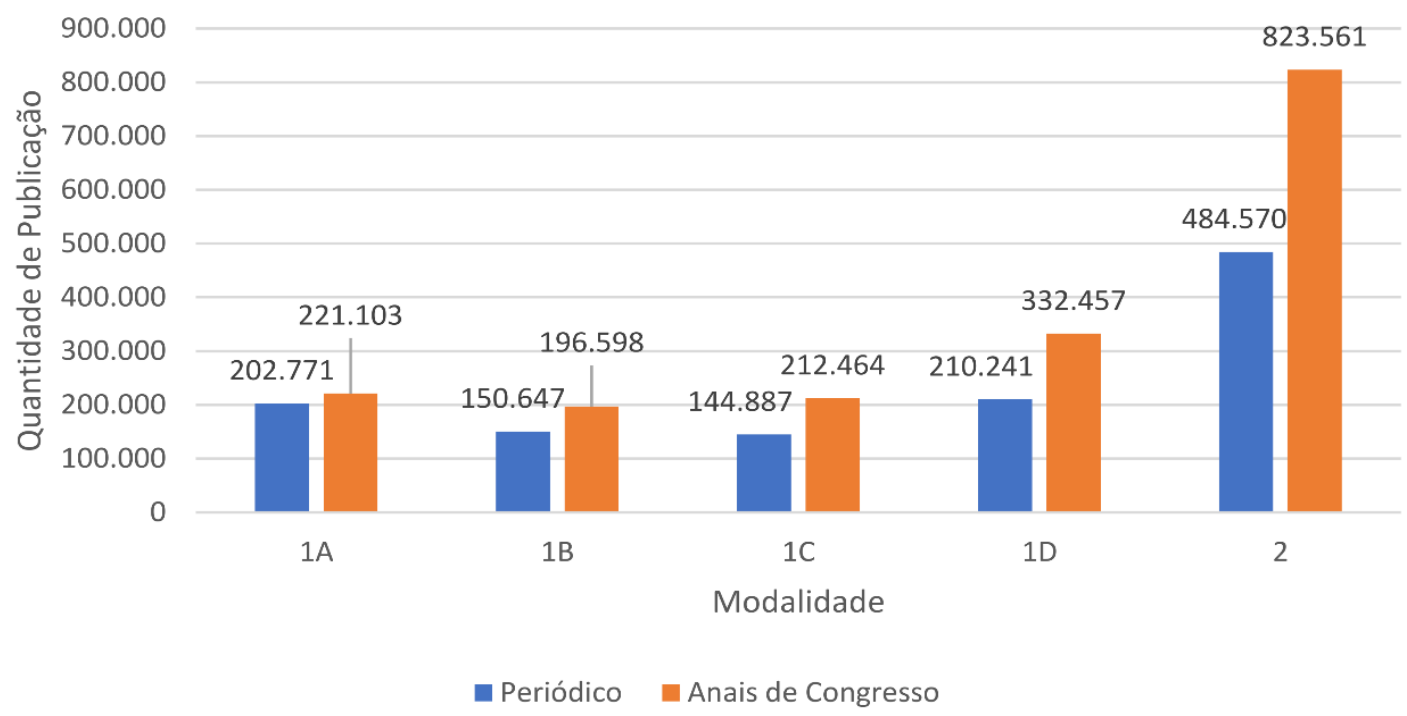

Figura 3. Total de publicações de artigos em anais de congressos e em periódicos

Ressalta que para a totalização das publicações em cada uma das modalidades, foram considerados todos os registros de publicações em cada um dos meios de divulgação encontrados nos currículos dos bolsistas, independentemente de quando o trabalho foi publicado.

Como pode ser observado, a modalidade 2 possui a maior média de publicações, tendo em vista que é a modalidade que contém a maior quantidade de bolsistas $(56,45 \%)$. A quantidade de publicações nas modalidades mais altas, tendem a se reduzir, já que concentram uma menor quantidade de bolsistas. Destaca-se, a semelhança entre a quantidade de publicações da modalidade $1 \mathrm{~A}$ com a $1 \mathrm{C}$. E ainda, nota-se que, à medida que o nível das modalidades de bolsas vai aumentando, a diferença entre publicações de artigos em anais de congressos e em periódicos tendem a reduzir.

Um tipo de análise que comumente vem sendo realizada quando verificada a produção científica de um determinado conjunto de indivíduos é a análise sobre como a comunidade científica tem colaborado (MENA-CHALCO; DIGIAMPIETRI; CESARJUNIOR, 2012; BOAVENTURA et al., 2014; MENA-CHALCO et al. 2014; DIGIAMPIETRI, 2015). Neste trabalho, são consideradas apenas as publicações de artigos em anais de congressos e em periódicos dos bolsistas para a caracterização das redes de colaboração científica a serem analisadas, já que são os principais meios de divulgação do conjunto.

Tais redes possibilitam analisar, com a adoção de métricas específicas, como as colaborações têm evoluído ao longo dos anos ou sobre como elas estão estruturadas. Neste trabalho, foram caracterizadas redes por modalidades de bolsas e, para suas análises, algumas métricas de análise de redes sociais foram aplicadas.

Para caracterização das redes que contêm todos os bolsistas, foram adotados métodos que visam concentrar os nós mais conectados no centro da rede e, consequentemente, aqueles nós com menor quantidade de ligações ou isolados são deslocados para as extremidades das redes (Figura 4). 


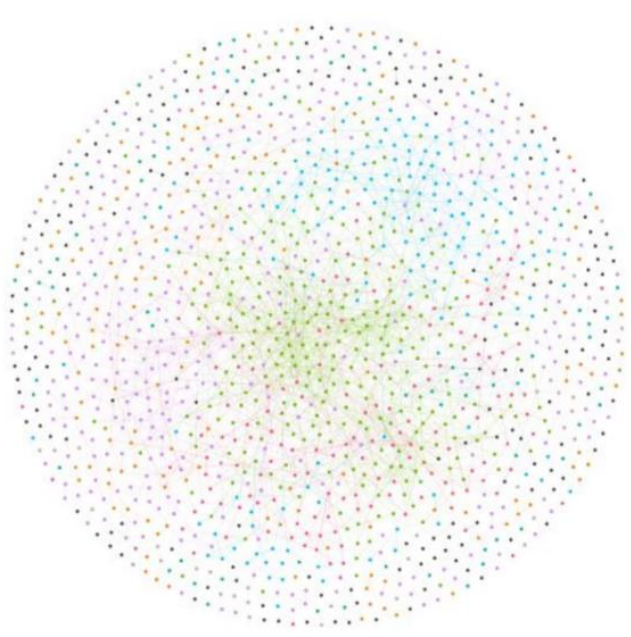

Modalidade da Bolsa - 1C

Modalidade da Bolsa - 1D

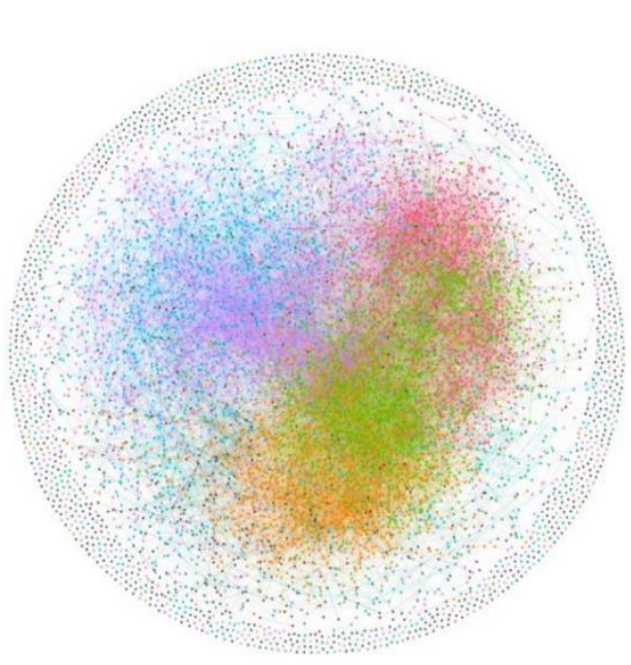

Modalidade da Bolsa - 2

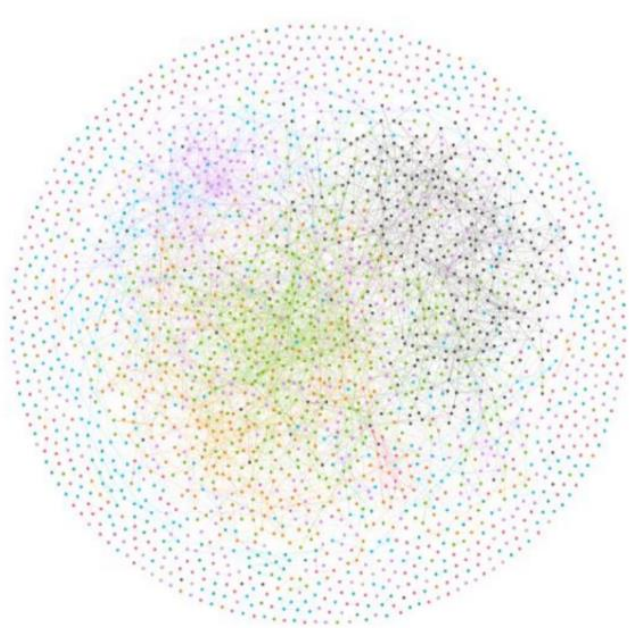

Ciências Agrárias

- Ciências Biológicas

- Ciências da Saúde

- Ciências Exatas e da Terra

- Ciências Humanas

- Ciências Sociais e Aplicadas

Engenharias

- Linguistica, Letras e Artes

- Outra

Figura 4. Redes de colaboração científica dos Bolsistas de Produtividade em Pesquisa do CNPq. 
Ao analisar as redes de colaboração de cada modalidade, é possível observar grande similaridade entre as redes das modalidades $1 \mathrm{~A}$ e $1 \mathrm{D}$, a exemplo do que ocorre com a produção científica. Em que suas componentes gigantes são maiores, e existe uma maior centralidade dos bolsistas agrupados principalmente, pelas suas grandes áreas. Já as redes das modalidades $1 \mathrm{~B}$ e $1 \mathrm{C}$, também são semelhantes, possuindo a menor quantidade de bolsistas nas suas componentes gigantes, tornando as redes mais esparsas. A rede da modalidade 2, que possui a maior quantidade de bolsistas, dentre todas as modalidades, se destaca por possuir a maior componente gigante, e ainda, a maior quantidade de componentes isolados, concentrados nas extremidades da rede. Agrupando as redes de todas as modalidades, é possível caracterizar a rede global (Figura 5).

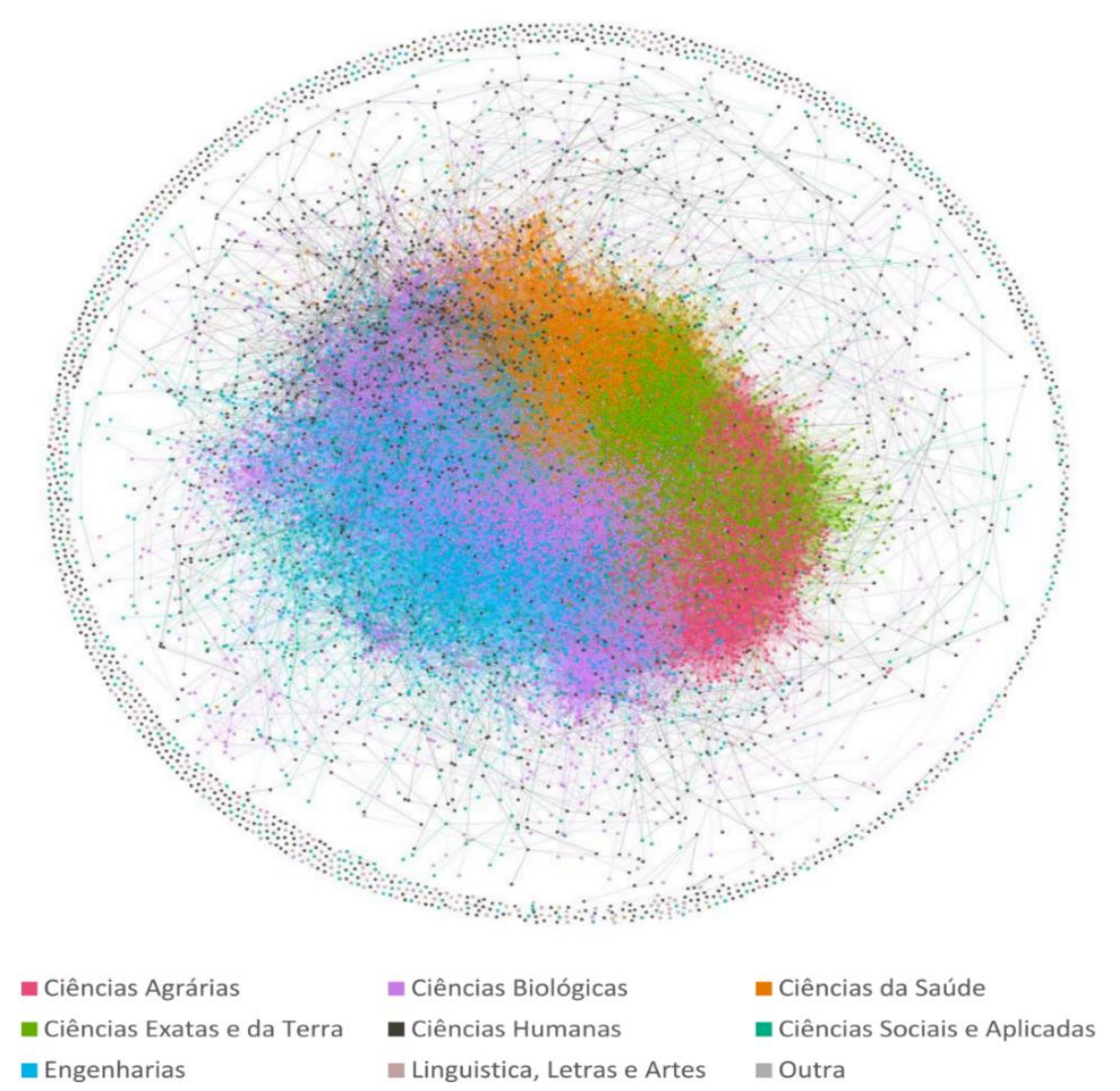
Figura 5. Rede global com todos os Bolsistas de Produtividade em Pesquisa do
CNPq

A rede global, contempla todo o conjunto de bolsistas de Produtividade em Pesquisa do $\mathrm{CNPq}$, possuindo a menor quantidade de nós isolados, que podem ser observados nas extremidades da rede, e sua componente gigante concentra conjuntos de bolsistas, agrupados principalmente pelas suas grandes áreas. No entanto, percebe-se considerável sobreposição de grandes áreas, o que representa colaboração entre bolsistas de grandes áreas distintas.

Após caracterizar as redes, algumas métricas foram aplicadas. As métricas adotadas (grau médio dos nós, total de nós no componente gigante, densidade da rede, diâmetro da rede e caminho mínimo médio) são métricas clássicas, usualmente adotadas por diversos trabalhos que analisam redes de colaboração (SZWARCFITER, 1986; NEWMAN, 2003; LEMIEUX; OUIMET, 2008; SCOTT, 2009; WASSERMAN; FAUST, 2009). 
A Tabela 1 apresenta uma sumarização das redes caracterizadas e das métricas adotadas em todos as modalidades de bolsas. Pode-se afirmar que a análise das redes de colaboração científica do conjunto de Bolsistas de Produtividade em Pesquisa do CNPq possibilita obter uma visão de como ocorreu o processo de coautoria entre os principais pesquisadores em atuação no Brasil.

Tabela 1. Resultado das métricas adotadas

\begin{tabular}{|c|c|c|c|c|c|c|c|c|c|c|}
\hline \multicolumn{11}{|c|}{ Métrica } \\
\hline $\begin{array}{l}\text { Nível do } \\
\text { Bolsista }\end{array}$ & 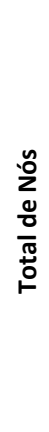 & 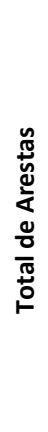 & 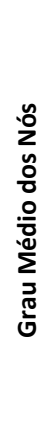 & 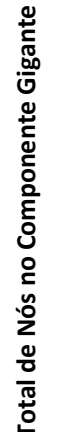 & 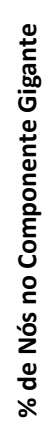 & 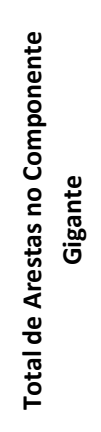 & 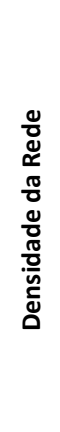 & 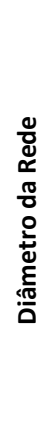 & 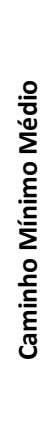 & 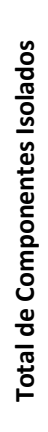 \\
\hline
\end{tabular}

\begin{tabular}{lllllllllll}
\hline $1 \mathrm{~A}$ & 1.180 & 5.402 & 9,15 & 884 & $74,92 \%$ & 5.293 & 0,008 & 15 & 4,74 & 202 \\
\hline $1 \mathrm{~B}$ & 1.267 & 3.156 & 4,98 & 835 & $65,90 \%$ & 3.024 & 0,004 & 19 & 5,64 & 304 \\
\hline $1 \mathrm{C}$ & 1.432 & 3.170 & 4,42 & 957 & $66,83 \%$ & 3.052 & 0,003 & 27 & 6,36 & 343 \\
\hline $1 \mathrm{D}$ & 2.425 & 6.399 & 5,27 & 1.707 & $70,39 \%$ & 6.113 & 0,002 & 21 & 6,03 & 467 \\
\hline 2 & 8.171 & 37.225 & 9,11 & 6.685 & $81,81 \%$ & 36.839 & 0,001 & 19 & 5,69 & 1.079 \\
\hline Geral & 14.608 & 218.788 & 14,95 & 13.375 & $91,56 \%$ & 218.564 & 0,002 & 18 & 4,37 & 987 \\
\hline
\end{tabular}

É possível observar a distinção entre as estruturas das redes, sendo que a rede global que comtempla todos os bolsistas, possui como era de se esperar, a maior componente conectada, concentrando $99,9 \%$ das arestas da rede e 91,56\% dos bolsistas. No entanto, ela possui menor densidade que algumas redes específicas, como por exemplo das modalidades 1A, 1B e 1C. Logo, podemos afirmar que, apesar da rede geral possuir a maior componente conexa, as redes de algumas modalidades específicas, são bem mais densas, resultado que nestas redes, os bolsistas estão mais conectados, necessariamente, nos níveis mais alto de modalidade das bolsas.

Ressalta-se ainda, o diâmetro da rede da modalidade 1A, o menor dentre todas as outras, resultando em um menor esforço para conectar os dois bolsistas mais distantes da rede. Valor este, bem inferior ao de outras modalidades, como por exemplo o da modalidade 1C, em que existe uma distância de 26 bolsistas entre os dois mais distantes.

Destaca-se a rede geral, que possui o maior valor do grau médio $(14,95)$, podendo ser considerada a rede de maior colaboração tendo em vista, que contempla todos os bolsistas. Foi possível ainda identificar que, apesar de as redes possuírem baixa densidade, as das modalidades de maior nível, possuem as menores quantidades de componentes isolados, que correspondem a autores que publicaram sem nenhuma colaboração ou que não publicaram com outros bolsistas de sua mesma modalidade.

Também foi possível verificar como alguns pesquisadores têm trabalhado em colaboração de forma muito intensa. Analisando as arestas mais densas das redes, alguns bolsistas se destacam com intensa quantidade de colaborações, em que para todas as 
redes, as arestas com maior peso possuem centenas de colaborações, resultado de intensa frequência na produção de artigos científicos. Em geral, tais colaborações acontecem em sua maioria na publicação de artigos em anais de congressos, e são entre pesquisadores que atuam na mesma área ou em áreas correlatadas, que possuem a mesma formação acadêmica e, em determinadas situações, orientados pelos mesmos orientadores.

Logo, as análises apresentadas possibilitam compreender como ocorre a colaboração científica entre os pesquisadores de excelência no Brasil, analisando todo o histórico de suas publicações científicas registradas nos currículos cadastrados na Plataforma Lattes.

\section{Considerações Finais}

Como resultado deste estudo, foi possível verificar como ocorre a colaboração científica do conjunto de Bolsistas de Produtividade em Pesquisa do CNPq. Para isso, foram considerados os artigos publicados em anais de congresso e em periódicos contemplando todo o seu histórico de publicações. Identificou-se que a produção cientifica dos bolsistas da modalidade 1A é distribuída quase que igualitariamente entre os dois tipos de publicações, diferentemente dos níveis mais baixos de modalidade das bolsas, em que as publicações são realizadas, em sua grande maioria em anais de congressos.

Foi possível observar que nas modalidades mais altas de bolsas, a colaboração ocorre de forma mais intensa, e que nos níveis mais inferiores a quantidade de bolsistas que não colaboram com bolsistas da mesma modalidade é bem superior. Com isso concluímos que os pesquisadores de excelência do Brasil, têm colaborado entre si, e que esta colaboração ocorre de forma mais intensa entre os bolsistas das modalidades superiores. Além disso, analisando outros dados dos bolsistas, foi possível perceber também que existe colaboração entre bolsistas de diferentes níveis de modalidades de bolsas, e que a colaboração é fortemente influenciada pela localização geográfica dos bolsistas, por exemplo, entre bolsistas de uma mesma instituição ou estado.

Como trabalhos futuros pretende-se verificar como é estruturada a rede de colaboração científica do conjunto de doutores com currículos cadastrados na Plataforma Lattes que não são bolsistas, e dessa forma, verificar de forma comparativa a colaboração dos pesquisadores de excelência com os pesquisadores que não recebem bolsa.

\section{Agradecimentos}

Os autores agradecem ao CEFET-MG e a UEMG pelo auxílio na pesquisa.

\section{Referencias}

Araújo, C. A. (2006). Bibliometria: evolução histórica e questões atuais. Em Questão, 12, pp. 11-32.

Barabâsi, A. L., Jeong, H., Néda, Z., Ravasz, E., Schubert, A., \& Vicsek, T. (2002). Evolution of the social network of scientific collaborations. Physica A: Statistical mechanics and its applications, 311, pp. 590-614.

Boaventura, M., Bonson, K., Silva, A. P., Veloso, A., \& Meira Jr, W. (2014). Caracterização Temporal das Redes de Colaboração Científica nas Universidades Brasileiras: Anos 20002013. In: Brazilian Workshop on Social Network Analysis and Mining. Brasília. 
Dias, T. M. R. (2016). Um Estudo Sobre A Produção Científica Brasileira A Partir De Dados Da Plataforma Lattes. Tese (Doutorado em Modelagem Matemática e Computacional) - Centro Federal de Educação Tecnológica de Minas Gerais, Belo Horizonte - MG.

Digiampietri, L. A. (2015). Análise da Rede Social Acadêmica Brasileira. (Livre Docência). Escola de Artes Ciências e Humanidades da Universidade de São Paulo, São Paulo.

Digiampietri, L., Mugnaini, R., Pérez-Alcázar, J., Delgado, K., Tuesta, E., \& Mena-Chalco, J. (2017). Aná lise da evolução, impacto e formação de redes nos cinco anos do BraSNAM. In Congresso da Sociedade Brasileira de Computação-CSBC.

Ding, Y. (2011). Scientific collaboration and endorsement: Network analysis of coauthorship and citation networks. Informetrics, 5, pp. 187-203.

Hoffnagel, J. C. (2009). A prática de citação em trabalhos acadêmicos. Cadernos de Linguagem e Sociedade, 10, p. 71.

Lane, J. (2010). Let's make science metrics more scientific. Nature, 464, pp. 488-489.

Lemieux, V., \& Ouimet, M. (2008). Análise estrutural das redes sociais. Lisboa: Instituto Piaget.

Lopes, G. R. (2012). Avaliação e Recomendação de Colaborações em Redes Sociais Acadêmicas. (Doutorado). Instituto de Informática UFRGS, Universidade Federal do Rio Grande do Sul, Porto Alegre.

Mena-Chalco, J. P., Digiampietri, L. A., \& Cesar-Junior, R. M. (2012). Caracterizando as redes de coautoria de currículos Lattes. In: Brazilian Workshop on Social Network Analysis and Mining. Curitiba.

Mena- Chalco, J. P., Digiampietri, L. A., Lopes, F. M., \& Cesar, R. M. (2014). Brazilian bibliometric coauthorship networks. Journal of the Association for Information Science and Technology, 65, pp. 1424-1445.

Mugnaini, R., Jannuzzi, P., Quoniam, L. (2004). Indicadores bibliométricos da produção científica brasileira: uma análise a partir da base Pascal. CI, 33, pp.123-131.

Newman, M. E. (2001). The structure of scientific collaboration networks. Proceedings of the National Academy of Sciences, 98, pp. 404-409.

Pritchard, A. (1969). Statistical bibliography or bibliometrics? Journal of Documentation, 4, pp. 348-349.

Revoredo, K., Araújo, R., Silveira, B., \& Muramatsu, T. (2012). Minerando publicações científicas para análise da colaboração em comunidades de pesquisa. Brazilian Workshop on Social Network Analysis and Mining (BraSNAM). Curitiba - PR.

Santos, L. R. F. (2015). Utilização de Dados da Plataforma Lattes para a Avaliação da Distribuição da Bolsa de Produtividade em Pesquisa do CNPq. Dissertação (Mestrado em Modelagem Matemática e Computacional) - Centro Federal de Educação Tecnológica de Minas Gerais, Belo Horizonte.

Scott, J. (2009). Social network analysis: a handbook (2 ed.). London: SAGE.

Silva, M. R., Hayashi, C. R., \& Hayashi, M. C. (2011). Análise bibliométrica e cientométrica: desafios para especialistas que atuam no campo. InCID: Revista de Ciência da Informação e Documentação, 2, pp. 110-129.

Sonnenwald, D. H. (2007). Scientific collaboration. Annual Review of Information Science and Technology, 41, pp. 643-681.

Ströele, V., Zimbrão, G., \& Souza, J. M. (2012). Análise de redes sociais científicas: modelagem multi-relacional. In: Brazilian Workshop on Social Network Analysis and Mining. Curitiba.

Szwarcfiter, J. L. (1986). Grafos e algoritmos computacionais (2 ed.). Rio de Janeiro: Campus.

Wasserman, S., \& Faust, K. (2009). Social network analysis: methods and applications (19 ed.). 